\title{
The Roll-out of Child-friendly Fixed-dose Combination TB Formulations in High-TB-Burden Countries: A Case Study of STEP-TB
}

\author{
Lena Faust ${ }^{1,2,}$, Kawsar Abdi ${ }^{3}$, Kristin Davis ${ }^{4}$, Chen $\mathrm{He}^{1, \dagger}$, Caitlin Mehrotra ${ }^{5}$, Emilie Stibolt ${ }^{6}$ \\ ${ }^{1}$ Department of Epidemiology, Biostatistics and Occupational Health, McGill University, Canada \\ ${ }^{2}$ McGill International TB Centre, Canada \\ ${ }^{3}$ School of Human Nutrition, McGill University, Canada \\ ${ }^{4}$ Department of Microbiology and Immunology, Faculty of Medicine, McGill University, Canada \\ ${ }^{5}$ Department of Pharmacology, McGill University, Canada \\ ${ }^{6}$ Department of Geography, McGill University, Canada
}

\section{ARTICLE INFO}

\section{Article History}

Received 11 April 2019

Accepted 07 August 2019

Keywords

Pediatric tuberculosis

fixed-dose combinations

impact evaluation

Kenya

STEP-TB

\begin{abstract}
Childhood tuberculosis (TB) has hitherto been treated through estimation of pediatric doses through the crushing of adult pills, but the bitter taste of the pills and the inaccuracy of this dosing method presents a challenge for both patients and healthcare providers, leading to poor treatment outcomes. The TB Alliance therefore launched the Speeding Treatments to End PediatricTuberculosis (STEP-TB) project to incentivize the introduction of pediatric Fixed-Dose Combinations (FDCs) of TB drugs. This case study describes the elements of this project, evaluates its impact, and highlights future challenges for pediatric TB treatment. The impact assessment incorporates both market impact as well as projected public health impact, evaluating the availability, affordability, and quality of the FDCs, and lastly providing a projection of lives saved as a result of scale-up of the FDCs to near-universal availability and utilization, based on a publicly available pediatric TB-specific model. STEP-TB resulted in the development of two child-friendly FDCs that were successfully brought to market and made available in 20 of the project's 22 high-burden countries. On the basis of a country-specific projection of pediatric TB mortality in Kenya, scale-up to nearuniversal availability and utilization of the new FDCs could reduce pediatric TB-associated mortality by 2660 cases over the next 5 years. Future challenges include maintaining affordable prices for the FDCs and considering mechanisms to incentivize their introduction among high-risk groups in low-burden countries.
\end{abstract}

(C) 2019 Atlantis Press International B.V.

This is an open access article distributed under the CC BY-NC 4.0 license (http://creativecommons.org/licenses/by-nc/4.0/).

\section{INTRODUCTION}

\subsection{Background}

Tuberculosis (TB) is an infectious disease caused by the bacterium Mycobacterium tuberculosis. Globally, TB is responsible for a greater number of deaths than any other single infectious disease, and an estimated $80 \%$ of the global burden of TB morbidity is attributable to 22 high-burden countries (HBCs) [1], eight of which are in Africa [2] (Nigeria, Ethiopia, South Africa, Kenya, DR Congo, Tanzania, Uganda, and Mozambique). Approximately $10 \%$ of TB patients are children [3], and the World Health Organization (WHO) estimates that more than 1 million children under 15 years of age will fall ill with active TB disease each year [1]. Unfortunately, addressing childhood TB has not been a priority in the past in comparison to $\mathrm{TB}$ in adults [1], and TB control in children is hindered by the fact that accurate diagnosis of TB in children remains a challenge.

"Corresponding author.Email: lena.faust@mail.mcgill.ca

'Lady Davis Institute for Medical Research, Jewish General Hospital, Canada Data availability statement: All data used in the impact projection of lives saved is publicly available through the MAP-IT Model Platform [7] (http://www.mapit4pedstb.org/).
Children are, however, at increased risk of progression to active TB disease, making accurate diagnosis and prompt treatment initiation crucial in this group [4]. Moreover, TB treatment in children is considered critical to the attainment of the Sustainable Development Goal of ending preventable deaths in children by 2030 .

In response to evidence that children require specific dosing of $\mathrm{TB}$ regimens to optimize treatment effect, the $\mathrm{WHO}$ revised its dosing guidelines for the treatment of childhood TB in 2010 [1], and called for "appropriately dosed, quality medicines in a childfriendly format"; however, regrettably, no interest was demonstrated by pharmaceutical companies [5]. Consequently, caregivers and healthcare providers have been obliged to rely on their best judgment in estimating doses, which involves splitting or crushing the bitter-tasting adult pills. This represents a tremendous challenge for both children and caregivers, leading to inaccurate dosing, poor compliance with the regimens, and ultimately poor treatment outcomes [5].

In 2012, Unitaid pledged US\$16.7 million to the Global Alliance for TB Drug Development (the TB Alliance), a not-for-profit drug development and delivery organization, to develop pediatric Fixed-Dose Combinations (FDCs) of existing TB drugs [3]. This commitment 
by Unitaid led to the Speeding Treatments to End Pediatric-TB (STEP-TB) project, which engaged with the pharmaceutical sector to develop the FDCs. The first nationwide product launch and rollout of the new FDCs was in Kenya in October 2016 [5]. As of 2017, more than 1300 Kenyan children have initiated treatment with the new FDCs since their rollout, which at the time represented $21 \%$ of pediatric cases of TB in the country [1]. Having recently reached 1 million treatment orders globally, the child-friendly FDCs are now (as of June 2019) available in 93 countries, which together represent three quarters of the global burden of pediatric TB [6].

\subsection{Goals of STEP-TB}

The goal of STEP-TB was to generate "improved access to correctly dosed, properly formulated, affordable, high-quality TB medicines for children" [3]. A large part of the project was, therefore, the establishment of a sustainable market for these new medications $[3,5]$. More specifically, the TB Alliance identified the following target project outcomes for STEP-TB:

- Developing appropriately formulated first-line pediatric TB medicines.

- Making affordable optimized first-line pediatric TB medicines available globally.

- Reducing or eliminating market barriers to the introduction of pediatric FDCs.

- Engendering increased commitment among countries to adopt the new FDCs.

- Delineating a pathway for the introduction of the FDCs.

\subsection{Description of Intervention}

Introducing new and improved pediatric $\mathrm{TB}$ medicines was the cornerstone of the STEP-TB project. To do this, the TB Alliance partnered with three pharmaceutical companies already manufacturing TB medicines [3,5]. In December 2015, MacLeods, an Indiabased pharmaceutical company manufacturing both new products and generics then developed two pediatric FDC pills (using the existing TB drugs Rifampicin, Isoniazid, and Pyrazinamide, formulated for pediatric dosing) (See Table 1), and was the only com-

Table 1 The recommended dosages of the new child-friendly FDCs based on, and compliant with, the 2010 WHO guidelines for pediatric TB treatment. The formulations are a combination of two or all three of the existing drugs Rifampicin (R), Isoniazid (H), and Pyrazinamide $(\mathrm{Z})$, and are taken in two phases, the intensive phase (2 months) and the continuous phase (4 months) [5].

\begin{tabular}{lcc}
\hline & \multicolumn{2}{c}{ Number of tablets } \\
\cline { 2 - 2 } Weight band (kg) & Intensive phase & Continuous phase \\
\cline { 2 - 2 } & RHZ 75/50/150 (mg) & RH 75/50 (mg) \\
\hline $4-7$ & 1 & 1 \\
$8-11$ & 2 & 2 \\
$12-15$ & 3 & 3 \\
$16-24$ & 4 & 4 \\
$25+$ & Adult doses recommended \\
\hline
\end{tabular}

pany able to get their medicines to market within the allotted time frame [5]. The new FDCs were ultimately available in the correct pediatric doses, palatable flavors, and were water-soluble for young children unable to swallow pills. In January 2016, the FDCs became globally available through the Stop-TB Partnership's Global Drug Facility for US\$15.54 for a 6-month course of treatment $[3,5]$.

The goals of STEP-TB included not only the production of childfriendly FDCs but also delineating a pathway for their effective introduction. Therefore, to raise awareness of the new childfriendly FDCs in advance of their first national rollouts, the TB Alliance launched the "Louder than TB" campaign on World TB Day 2016 to position TB as a critical item on the child health and survival agenda, mobilize demand, and ensure there was a sufficient customer base for the medicines [5]. In October 2016, Kenya became the first country to rollout the new FDCs on a national scale [5]. The TB Alliance and Kenya's National Tuberculosis, Leprosy, and Lung Disease Program worked closely to organize a public launch followed by a sustained outreach campaign in support of the rollout [5]. In India, the STEP-TB project engaged with the national TB program and private providers, with the goal of supporting policy changes to ensure access to treatment [5]. These HBC-focused approaches were designed to maximize the global outreach of the program, and they provided information regarding use of the FDCs, contributed to policy recommendations, and worked to ensure a quick and sustainable uptake of the new medicines $[3,5]$.

\section{MATERIALS AND METHODS}

\subsection{Impact Evaluation}

The key achievement of the STEP-TB project was incentivizing the market for - and ultimately making available - two new firstline child-friendly FDCs (RH 75/50 mg and RHZ 75/50/150 mg) [1]. To assess the impact of the program, we utilize the goals set by STEP-TB itself as a framework for initial impact evaluation, and lastly, we employ a pediatric TB-specific model [7] to provide a projection of the impact of near-universal utilization of the new pediatric FDCs on lives saved in Kenya, the first country to have rolled out the regimens [3].

\subsubsection{Framework for impact evaluation}

The self-articulated goals of the STEP-TB project were (1) to develop child-friendly, appropriately formulated medicines for the treatment of drug-susceptible TB and (2) to make these formulations available and affordable to children globally [1]. In terms of impact evaluation, the first of these goals raises the question of quality of the new FDCs, whereas the second relates to availability and affordability. Our initial impact evaluation of STEP-TB is therefore based on these three elements, aiming to determine the quality, availability, and affordability of the STEP-TB FDCs. Other outcomes relevant to the impact evaluation of the program, including more downstream measures of success, such as the potential impact of improved adherence and improved treatment outcomes with the new FDCs, could not be assessed in the current paper owing to the lack of patient-level data on these outcomes with the new FDCs [1]. 


\subsubsection{Projection of lives saved under conditions of near-universal availability and utilization of pediatric FDCs in Kenya}

The Model for Assessment of Pediatric Interventions for Tuberculosis (MAP-IT) was developed as part of the STEP-TB project [7]. The model allows estimation of lives saved through modification of different screening, diagnostic, or treatment parameters in relation to pediatric TB compared with baseline values estimated from country-specific data. We therefore used this model to estimate lives saved over the next 5 years if the availability and correct use of the new FDCs were to be scaled up to near-universal levels (defined by the model developers as 98\%) [7] in Kenya, the first country to have rolled out the pediatric FDCs. It is hypothesized that scale-up of availability and correct use of the child-friendly regimens may improve treatment outcomes owing to fewer dosing errors and higher adherence, which are otherwise significant barriers to the successful treatment of pediatric TB.

The time frame of the model covers the 5-year period from 20192024. As we aimed to estimate the impact of scale-up of the availability and use of the FDCs to near-universal levels (in public and private sector settings), the only parameters modified from their baseline values in the projection were (1) presumptive treatment for drug-susceptible TB and (2) clinical treatment for confirmed drug-susceptible TB. Further parameters in the model reflect standard screening, immunization, and diagnostic practices in Kenya, which were kept the same in both the baseline and comparator scenarios. Kenya-specific estimates for likelihood of progression to active disease and mortality are used to estimate lives saved.

\section{CASE STUDY RESULTS}

\subsection{Impact Evaluation}

\subsubsection{Quality}

Owing to the lack of individual-level data regarding the efficacy of the regimens in terms of improved pediatric TB treatment outcomes, WHO prequalification of the FDCs is the quality indicator for the purpose of this impact evaluation. Having first endorsed the FDCs in March 2017, the WHO and UNICEF released a joint statement urging "all national TB programmes to discontinue and replace the previously used medicines for children weighing $<25 \mathrm{~kg}$ with the childfriendly dispersible TB FDCs as soon as possible" [4]. Moreover, the new child-friendly FDCs are now included on the most recent iteration of the WHO List of Essential Medicines for Children, whereas previous FDCs are not $[1,5]$. Finally, WHO prequalification of the FDCs was obtained in September 2017, attesting to the quality of the regimens, although concrete measurements of quality (i.e., analyses of treatment success rates or adverse events) are not possible at this time given the absence of data on patient outcomes with the new FDCs [3].

\subsubsection{Availability}

Given that the key goals and strategy of the STEP-TB project centered around market incentivization and making the developed
FDCs widely available, availability in the 22 target countries, as well as global availability, is an important impact evaluation consideration. At the time of the final STEP-TB evaluation report (May 2017), 20 of the 22 HBCs had strategies for the introduction of the new formulations and for the phaseout of the old formulations, with only Russia and China not planning to introduce the FDCs [1,5] (Note that for the period of 2016-2020, 30 countries are classified as high TB-burden countries by the WHO, however, the STEP-TB project targeted the previously recognized $22 \mathrm{HBCs}$ [1].). Figures 1 and 2 show the order and availability status of the FDCs in the 22 target countries and the global uptake of the FDCs, respectively.

\subsubsection{Affordability}

In comparison to previous FDCs, the new pediatric FDCs are comparably priced, with a full course of treatment costing US\$15.54 (price range of previous FDCs: US\$13.55-22.00), and further price reductions are expected in the future $[1,3]$.

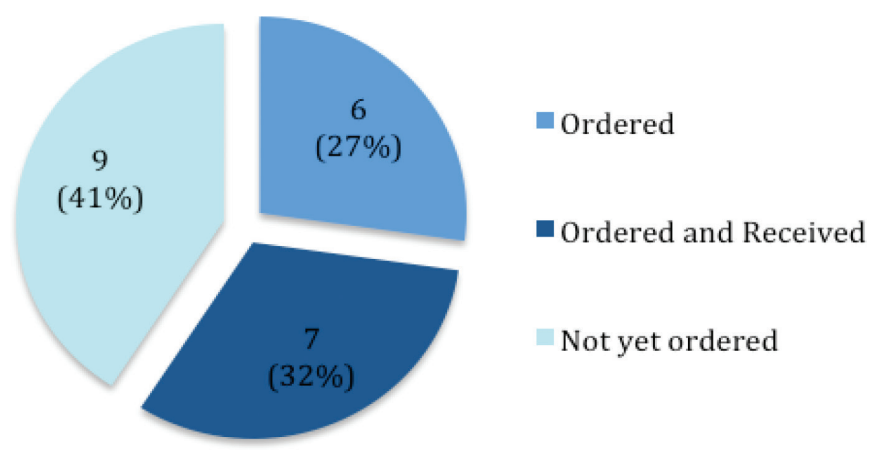

Figure 1 Availability of new pediatric FDCs (RH 75/50 mg and RHZ $75 / 50 / 150 \mathrm{mg}$ ) in the 22 high-burden target countries of STEP-TB [ $n(\%)]$, as of the final STEP-TB evaluation report (May 2017) [1].

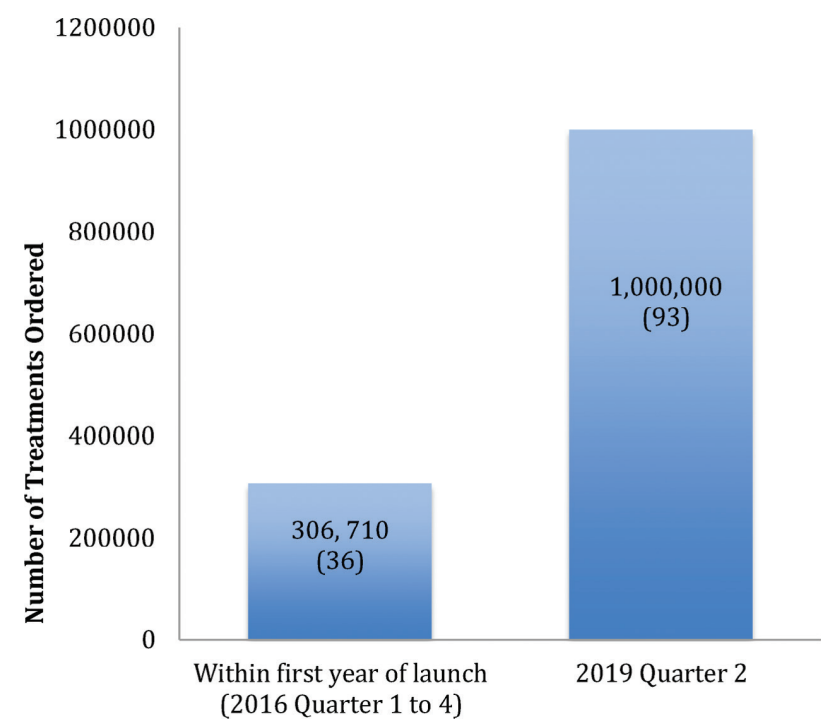

Figure 2 Global uptake of new child-friendly FDCs within the first year of the launch of STEP-TB (end-2016) compared with most recent estimates (mid-2019), by number of treatment courses ordered and number of countries ordering $[3,4,6]$. 


\subsubsection{Lives saved in Kenya: an impact projection of pediatric FDCs using the MAP-IT model: model parameters and assumptions}

As described in Subsection 2.1.2, the only parameters modified from their baseline values in the projection were (1) presumptive treatment for drug-susceptible TB and (2) clinical treatment for confirmed drug-susceptible TB (as the FDCs are currently only available for the treatment of drug-susceptible TB). Further parameters in the model represented standard screening, immunization, and diagnostic practices in Kenya, which were kept the same in both the baseline and comparator scenarios. These baseline parameters and assumptions are outlined in Table 2, and the modifications made to the availability (penetration) and utilization values for the FDCs in the comparator scenario vs. the baseline are shown in Table 3. We use the "moderate" estimation mode recommended by the model developers, which uses the midpoint range of confidence intervals for estimates, thereby taking into account the variability in the available data concerning intervention effects, TB incidence, and mortality rates [7].

\subsubsection{Impact projection results}

The results of the projected number of pediatric lives saved in Kenya over the 5-year period (2019-2024) under conditions of

Table 2 Baseline MAP-IT model parameter values for pediatric TB prevention, diagnosis, and treatment (with pediatric FDCs) in Kenya

\begin{tabular}{|c|c|c|c|c|c|}
\hline \multirow{2}{*}{\multicolumn{3}{|c|}{ Model parameters }} & \multicolumn{3}{|c|}{ Healthcare setting } \\
\hline & & & \multirow{2}{*}{$\begin{array}{c}\text { Community (\%) } \\
90\end{array}$} & \multirow{2}{*}{$\begin{array}{c}\text { Public (\%) } \\
90\end{array}$} & \multirow{2}{*}{$\begin{array}{c}\text { Private (\%) } \\
90\end{array}$} \\
\hline Preventive & BCG vaccination & Penetration & & & \\
\hline strategies & & Utilization & 85 & 85 & 85 \\
\hline & & Efficacy & 70 & 70 & 70 \\
\hline & Preventative treatment (e.g., IPT) & Penetration & 10 & 95 & 95 \\
\hline & & Utilization & 5 & 6 & 6 \\
\hline & & Efficacy & 35 & 35 & 35 \\
\hline \multirow[t]{18}{*}{ Diagnostics } & Tuberculin skin test & Penetration & 25 & 95 & 95 \\
\hline & & Utilization & 5 & 5 & 10 \\
\hline & & Efficacy & 50 & 50 & 50 \\
\hline & Identification of suspected active TB case through & Penetration & 25 & 0 & 0 \\
\hline & contact investigation & Utilization & 25 & 0 & 0 \\
\hline & & Efficacy & 84 & 84 & 84 \\
\hline & Clinician-recognized symptoms of active TB & Penetration & 50 & 95 & 95 \\
\hline & & Utilization & 20 & 50 & 50 \\
\hline & & Efficacy & 84 & 84 & 84 \\
\hline & Microbiology for active TB & Penetration & 5 & 90 & 90 \\
\hline & & Utilization & 5 & 50 & 50 \\
\hline & & Efficacy & 65 & 65 & 65 \\
\hline & Xpert for active TB & Penetration & 0 & 30 & 15 \\
\hline & & Utilization & 0 & 30 & 30 \\
\hline & & Efficacy & 80 & 80 & 80 \\
\hline & Radiology for active TB & Penetration & 0 & 50 & 50 \\
\hline & & Utilization & 0 & 20 & 15 \\
\hline & & Efficacy & 50 & 50 & 50 \\
\hline \multirow[t]{12}{*}{ Treatments } & Presumptive treatment for drug-sensitive TB with & Penetration & 10 & $90^{*}$ & $90^{*}$ \\
\hline & pediatric FDCs & Utilization & 10 & $40^{*}$ & $40^{*}$ \\
\hline & & Efficacy & 95 & 95 & 95 \\
\hline & Presumptive treatment for drug-resistant TB & Penetration & 10 & 90 & 90 \\
\hline & & Utilization & 10 & 20 & 20 \\
\hline & & Efficacy & 80 & 80 & 80 \\
\hline & Clinical treatment for confirmed drug-sensitive & Penetration & 10 & $90^{*}$ & $90^{*}$ \\
\hline & TB with pediatric FDCs & Utilization & 15 & $65^{*}$ & $65^{*}$ \\
\hline & & Efficacy & 95 & 95 & 95 \\
\hline & Clinical treatment for confirmed drug-resistant TB & Penetration & 10 & 90 & 90 \\
\hline & & Utilization & 25 & 60 & 60 \\
\hline & & Efficacy & 80 & 80 & 80 \\
\hline \multirow[t]{2}{*}{ Settings } & Location where children contract TB (i.e., latent TB) & & 98 & 1 & 1 \\
\hline & Location where children with active TB seek care & & 35 & 60 & 5 \\
\hline \multirow[t]{2}{*}{ Transfers } & Location where suspected cases of active TB are & & Home to clinic (\%) & Home to hospital (\%) & Clinic to hospital (\%) \\
\hline & identified through contact tracing seek care & & 65 & 5 & 0 \\
\hline
\end{tabular}

"Parameters indicated by an asterisk are those whose values were modified in the comparator scenario, with their modified values as shown in Table 3 . All other parameters listed above

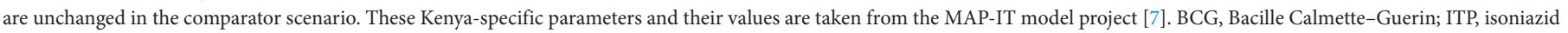
preventive therapy; Penetration, Availability; Utilization, Correct Use. 
Table 3 Modified parameter values for comparator scenario of near-universal penetration and utilization of pediatric FDCs (compared with MAP-IT model baseline values)

\begin{tabular}{|c|c|c|c|c|c|}
\hline \multirow[t]{2}{*}{ Model parameter } & & \multicolumn{2}{|c|}{ Baseline scenario } & \multicolumn{2}{|c|}{$\begin{array}{l}\text { Modified scenario for near-universal } \\
\text { penetration and utilization }\end{array}$} \\
\hline & & Public (\%) & Private (\%) & Public (\%) & Private (\%) \\
\hline \multirow{2}{*}{$\begin{array}{l}\text { Presumptive treatment for drug-sensitive TB with } \\
\text { pediatric FDCs }\end{array}$} & Penetration & 90 & 90 & 98 & 98 \\
\hline & Utilization & 40 & 40 & 98 & 98 \\
\hline \multirow{2}{*}{$\begin{array}{l}\text { Clinical treatment for confirmed drug-sensitive TB } \\
\text { with pediatric FDCs }\end{array}$} & Penetration & 90 & 90 & 98 & 98 \\
\hline & Utilization & 65 & 65 & 98 & 98 \\
\hline
\end{tabular}

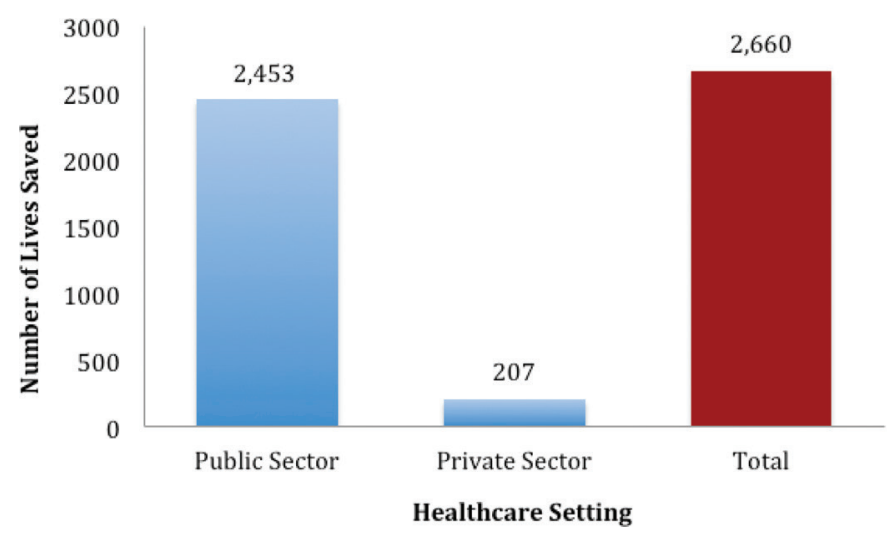

Figure 3 Estimated number of lives saved between 2019 and 2024 due to reductions in TB-associated mortality among children (0-14 years) in Kenya under near-universal availability and utilization of new child-friendly FDCs in the public and private sectors (assuming standard Kenyan screening, immunization, and diagnostic conditions). Estimates generated using the MAP-IT Model Version 1.0.2, Data version 3.0.0 (RTI International, North Carolina, USA) [7].

near-universal availability and utilization of the new FDCs is shown in Figure 3. Our projection suggests that if the availability and utilization of the new child-friendly FDCs in Kenya were to be scaled up from their current levels to near-universal levels (98\%) in the private and public sectors, 2660 lives could be saved between 2019 and 2024.

\subsection{Financing}

The STEP-TB project was launched through an investment of US\$ 16.7 million by Unitaid. Additional support from donors (such as USAID and the Global Fund), commercial partners, policy makers, and NTPs led to better implementation of newer policies, allowed for product-transition planning, better implementation of new registration strategies, rapid uptake, and affordable price negotiations [3,5]. Manufacturers were also offered financial compensation (amounting to US $\$ 1.5$ million) as an incentive to meet target deadlines, however, market demand was considered the main factor in engaging manufacturers. In addition, manufacturers were given subsidies to offset manufacturing costs, thereby keeping prices low [3]. Unfortunately, however, as mentioned earlier, only one manufacturer, MacLeods, ultimately entered the market. This is identified by STEP-TB as a major shortcoming, as the involvement of multiple manufacturers was a key aim of the project, and the failure to attain it represents a significant threat to keeping drug prices low in the long term through competition between multiple manufacturers.

Apart from the initial financial considerations involved in bringing a product onto the market, the financial challenges of scale-up must also be considered. To achieve scale-up, manufacturers first have an interest in ensuring that there will be a predictable and potentially growing market for the product [5]. One of the elements of STEP-TB's market research strategy was therefore to provide an estimate of the pediatric TB burden, which was hitherto not precise [3]. This allowed for more reliable estimation of the market size, which manufacturers were able to use to forecast sales and their Return on Investment (ROI).

A second element of successful scale-up is the retention of highvolume countries (those that have high demand for the product) in the market, allowing companies to attain ROI. The STEP-TB project facilitated this retention among HBCs through their work with NTPs, providing information on the appropriate use of the FDCs and engaging countries through the awareness campaign launched as part of the project [5].

\subsection{Drivers of Success}

Several reasons are attributable to the considerable success of the STEP-TB project. First, updated surveillance and modelling studies conducted as part of STEP-TB accurately assessed the magnitude of the childhood TB burden, providing the groundwork to make a case for the introduction of pediatric FDCs. With a more reliable estimate of the burden of pediatric TB, the TB Alliance generated a broad and valuable partner landscape involving academics, governments, non-governmental organizations, policy makers, and most importantly, the pharmaceutical and manufacturing industries, thus incentivizing the development of pediatric TB formulations, and uniting the previously fragmented and stagnant pediatric TB treatment landscape [3].

Also, contributing to the success of STEP-TB was the fact that the new formulations were made child-friendly to help facilitate that children take the drugs for the entire treatment course. This included dispersibility of the drugs, providing ease of administration for both the medical personnel and children. Likewise, the improved taste and palatability has the potential to improve treatment adherence among children, which has been a persistent challenge in pediatric TB treatment [3]. Also, in contrast to the previous haphazard dosage estimates, the optimized pharmacodynamics of the new pediatric FDCs have the potential to markedly improve treat- 
ment outcomes [3]. Another significant driver of success was, and continues to be, the affordability of the child-friendly formulations, with a 6-month course costing approximately US $\$ 15.45$ being within range of affordability [1,3].

Lastly, several strategic elements of the STEP-TB program helped facilitate the national rollout of the FDCs, such as the launching of the "Louder than TB campaign" in Kenya to raise awareness of pediatric TB prior to the rollout of the FDCs [3], and the planned phasing out of the existing old formulations while waiting for the new FDCs to become available [3].

\section{DISCUSSION}

\subsection{Limitations of Program Impact}

Despite their inclusion on the WHO List of Essential Medicines for Children, their prequalification by the WHO, and their availability through the Global Drug Facility, regulatory barriers continue to hinder the adoption of the new FDCs, particularly in lowburden countries. In the European Union, for example, childfriendly FDCs are not registered with the necessary regulatory agency (the European Medicines Agency) due to low market incentives for the formulations in this region [4]. The continued lack of incentivization for the introduction of pediatric formulations in low-burden countries, and the barriers this represents for highrisk groups in these countries are also exemplified by the lack of access to pediatric FDCs in Canadian indigenous communities [8]. Although low-burden countries were not included in STEP-TB's initial 22 target countries, STEP-TB did articulate an overall goal of incentivizing global access to pediatric FDCs, so barriers to access in Europe and other low-burden countries remains a relevant limitation of its impact. The STEP-TB's strategy of market incentivization to generate access to child-friendly FDCs has thus had significant impact in HBCs, but is less successfully applicable to the context of high-risk populations in low-burden countries, and thus represents a failure of the program with regard to paving the way for global availability of child-friendly TB treatment.

\subsection{Limitations of Impact Evaluation}

Apart from considering the limitations of actual project impact, the limitations of the impact assessment must also be recognized. There continues to be uncertainty surrounding the accuracy of estimates of the burden of childhood TB due to difficulties of diagnosis in children, and a historical lack of prioritization of pediatric TB limits the comprehensive assessment of the impact of pediatric TB interventions. The uncertainty in these estimates is also a limitation of the MAP-IT model [9], and the results of the impact projection should therefore be interpreted keeping in mind the variability in the estimates on which this projection is based [7]. An additional limitation of the model is that, given the lack of individual-level data on the efficacy of the pediatric FDCs, this parameter is not yet accurately reflected in current projections of lives saved, and no causal claims can be made regarding the implementation of the FDCs and improved treatment outcomes.
Further barriers to accurately assess the project's public health impact include the fact that, although information on order volumes is available, high-order volumes do not guarantee high coverage or appropriate use, and consequently, availability at the NTP level does not necessarily reflect actual access at the patient level [1]. Moreover, given the lack of data on improved adherence to the new FDCs or improved treatment outcomes with the new FDCs (vs. custom titration), the ultimate public health impact of the rollout of these new pediatric formulations in terms of improved treatment outcomes and reduced TB-associated mortality could not be directly assessed in this impact evaluation.

\subsection{Future Directions and Challenges}

The future considerations of the STEP-TB project include continuing to ensure access to the FDCs, to reshape the market for pediatric TB drugs, and to reduce barriers to market entry. To achieve these goals, sustainability is key [1]. First, a sustainable market is necessary for ensuring continued affordable access to the products. Also, necessary are sustainable partnerships with multi-stakeholders such as the academic community, clinicians, manufacturers and donor agencies, non-profit organizations, governments, policy makers, and regulatory authorities. Subsequently, sustainable integration and collaboration with low- and middle-income, high-TB burden countries is required, as is addressing the neglected position of select high-risk groups in otherwise low-burden countries in terms of the market landscape of pediatric TB formulations [10]. Challenges that persist for STEP-TB to address therefore include: maintaining sustained interest from partners, data transparency, the lack of patient-level data on actual treatment outcomes with the new FDCs, which limits impact assessment, and the lack of childfriendly formulations to treat drug-resistant forms of TB [1].

\section{CONCLUSION}

This case study provides a descriptive overview of the key strategies of STEP-TB, and an assessment of its impact, including a projection of lives saved as a result of scale-up of the FDCs to near-universal availability and utilization in Kenya. Although our projection indicates that near-universal availability and utilization of the new FDCs could reduce pediatric TB-associated mortality in Kenya by 2660 cases over the next 5 years, the results of this case study are substantially limited by the lack of individual patientlevel data on the efficacy of the new pediatric FDCs, which prevents a detailed quantitative analysis of the public health impact of the STEP-TB program. The program successfully incentivized the introduction of pediatric FDCs to HBC markets; however, ongoing challenges include maintaining affordable prices for the FDCs (particularly given the potential for monopoly due to current production by a sole manufacturer). In addition, there remains a need for mechanisms to incentivize the introduction of the FDCs for high-risk groups in low-burden countries, which is a need not yet sufficiently addressed by the STEP-TB's current market incentivization strategy. Lastly, the development of child-friendly formulations for drug-resistant TB is another remaining challenge. 


\section{CONFLICTS OF INTEREST}

The authors declare they have no conflicts of interest.

\section{AUTHOR CONTRIBUTIONS}

L.F. was responsible for quantitative aspects of the impact evaluation and initial drafting of the manuscript, and K.A., K.D., C.H., C.M. and E.S. were responsible for descriptive aspects of the impact evaluation. All authors read and approved the final manuscript.

\section{FUNDING}

None.

\section{ACKNOWLEDGMENTS}

The authors gratefully acknowledge Ms. Sarah Cook-Scalise and Ms. Shelly Malhotra at the TB Alliance for the valuable insights and expertise they contributed to this case study.

\section{REFERENCES}

[1] World Health Organization. Best practices in child and adolescent tuberculosis care. 2018. Available from: https://www.who.int/tb/ publications/2018/best-practices-child-adolescent-TB-care/en/

[2] World Health Organization Strategic and Technical Advisory Group for TB (STAG-TB). Use of high burden country lists for TB by WHO in the post-2015 era. 2015. Available from: https:// www.who.int/tb/publications/global_report/high_tb_burdencountrylists2016-2020.pdf
[3] Bourdin Trunz B, Brouwer M. Global Alliance for TB drug development (TB Alliance) speeding treatments to end paediatric tuberculosis (STEP-TB) end of project evaluation. 2017. Available from: https://unitaid.org/assets/UNITAID-STEPTBFinal-Evaluation-04July2017.pdf

[4] Onyango DO, Yuen CM, Masini E, Borgdorff MW. Epidemiology of pediatric tuberculosis in Kenya and risk factors for mortality during treatment: a national retrospective cohort study. J Pediatr 2018;201;115-121.

[5] New pathways for childhood TB treatment - Lessons from the STEP-TB project. TB Alliance, Unitaid. 2017. Available from: https://www.tballiance.org/sites/default/files/child-resources/ New_Pathways_for_Childhood_TB_Treatment.pdf

[6] Global Alliance for TB drug development (TB Alliance). New milestone for children with tuberculosis reached as one million treatments of child-friendly medicines are ordered. 2019. Available from: https://www.tballiance.org/news/one-million-child-friendly-tuberculosis-medicines

[7] RTI International. Model for assessment of pediatric interventions for tuberculosis (MAP-IT). 2015. Available from: http:// www.mapit4pedstb.org/

[8] Nash M. Making the case for child-friendly TB treatment for Inuit: challenging Canada's double standard. 2018. Available from: https://naturemicrobiologycommunity.nature.com/users/ 72229-madlen-nash/posts/40701-making-the-case-forchild-friendly-tb-treatment-for-inuit-challenging-canada-sdouble-standard

[9] World Health Organization. WHO model list of essential medicines for children: 6th list. 2017. Available from: http://apps.who. int/iris/bitstream/handle/10665/273825/EMLc-6-eng.pdf?ua=1

[10] Medecins sans Frontieres \& Stop TB Partnership. Out of step 2017 - TB policies in 29 countries: a survey of prevention, testing and treatment policies and practices. 2017. Available from: http:// www.stoptb.org/assets/documents/outofstep/UNOPS_out_of_ step_2017_55_online.pdf 